\title{
PINEAL HORMONE MELATONIN AS UNIVERSAL MODULATOR OF ANY PATHOLOGICAL PROCESSES
}

\author{
Arushanyan E. B. , Shchetinin E. V.
}

\section{Stavropol State Medical University, Russian Federation}

T: he Department of Pharmacology at the Stavropol State Medical Institute (later renamed as Academy and University) was among the first ones in our country, starting in 1983 , toconductacomplexstudyofthebiological properties and therapeutic possibilities of melatonin (MT), which was identified as a main hormone of the pineal gland. Own 30year experience of investigations coupled with much recent literary data, which was bellow summarized, allows so untraditional formulated problem in title this article. Unfortunately, the limited its volume oblige sending for concrete references to some reviews.

\section{Nature and biological (adaptogenic) role of $\mathrm{MT}$}

MT obtained from pineal glands of animals only in middle of $X X$ century to be later identified as its specific and main hormone with very large biological properties. This information has been repeatedly summarized in series reviews before [1, $6,68]$, which allows selecting some key points.

Synthesis of MT with an indole structure goes through several stages in the pineal cells pinealocytes. The initial part is tryptophan, which through serotonin and $\mathrm{N}$-acetylserotonin - turns into the end hormone. The resulting MT secretes into neurolymph further going into the bloodstream, after which the bloodflow takes it through the entire in different organs. Its effects come out in various brain structures and in peripheral tissues through specific receptors (mostly MT1 and MT2 Type). With no regard to the animal species, the development of pineal MT is at maximum in the dark and minimum in the light part of the day. This allows considering pineal as important chronotropic gland involved into night sleep regulation and circadian fluctuations of many physiological functions. As a result, MT is known as a natural hypnogenic agent and biological clock regulator - a chronobiotic, which obtained universal adaptogenic properties.

Arushanyan Eduard, MD, PhD, Professor, Head of Department of Pharmacology, Stavropol State Medical University;

tel.: (8652)354881; e-mail: eduard.arush@mail.ru

Shchetinin Eugeny, MD, PhD, Head of Department of Pathological Phisiology, Stavropol State Medical University;

tel.: (8652)352524; e-mail: ev.cliph@rambler.ru
The points in question were defined at the initial stage of studying the physiology of pineal and its biologically active compounds. In the meantime, in the 1970s already the existing immunehistochemical technique allowed detecting, along with the pineal MT, another independent MT in many peripheral cells and tissues (retina, gastrointestinal tract, blood cells, etc.). Just like in pineal gkand, this MT goes through similar synthesis stages involving similar enzymes. Interesting to note that the total amount of MT in tissues of the secreting organ may, by many times exceed, exceed the level of pineal-produced plasma hormone. This extreme prevalence made it possible to suggest that peripheral MT, similarly the central (pineal) one may have a key role as the paracrine signal molecule for the regional coordination of cell functions [34, $44,54]$. Due to its high lipophily, the MT circulating in blood, acting as a typical hormone, reaches the remote target cells and, through a different mechanisms (see below) provides their universal protection against any pathogenic processes.

\section{Protective effect of MT in organ pathology \\ Functional and organic brain disorders}

Proceed earlier detected capacity of MT to reduce behavioral and EEG manifestations of insomnia, late appeared some evidences of its positive influence on the psychoemotional states both in animals and human. In particular, after acute and especially chronic administration of even low MT doses $(0.1-1 \mathrm{mg} / \mathrm{kg})$ into rats usual behavior tests (open field test, elevated plus-maze or radial tests, etc.) showed its suppressive effect on the animals' fear and anxiety. There was also a clearly attenuated of behavioral disturbances due to various exogenous and endogenous stressors, while the people demonstrated significantly lower level of anxiety. On this criteria MT may be considered an antistressor drugs similar to benzodiazepine anxiolytics, at the same time standing rather close to these following their psychotropic activity range. Even earlier, such facts already allowed considering pineal as organ involved into global protection of brain against any form of stress $[2,4]$.

Given that, MT proved able to limit affective disorders as well. Its antidepressant effect was demonstrated on various experimental models of psychical depression. This fact was first shown 
in our laboratory while evaluating the time course of forced swimming in rats. The MT (1-2 mg/ $\mathrm{kg}$ ) did not only limit their immobility periods in water (viewed as behavioral despair), but also decreased chronobiological index of depression. Simultaneously it weakened the effect of a some of depressogenic agents (reserpine, clonidine) [31].

That knowledge helped us formulate an idea of considering MT as a natural antidepressant/ This approach which found no support in psychopharmacology once, however, later group of French investigates based on own results obtained new so called melatoninergic antidepressant valdoxan with high therapeutic potential. Unlike from traditional antidepressant drugs it not influenced on central monoaminergic transmission, but selectively stimulates certain types of MT and serotonin receptors [10].

Another type of psychotropic activity discovered in $\mathrm{MT}$ is manifested in its activation of cognitive functions seen as improved memory and learning. This is illustrated through rodent models evaluating the above-mentioned functions after they were disturbed under immobilization stress or in case of using amnesic agents. Such animals have their shortterm memory affected (evaluated with conditioned passive avoidance in shuttle box or Morris water maze) as well as their learning processes. Repeated administration of MT $(0.5-1 \mathrm{mg} / \mathrm{kg})$ shortens the avoidance response latency and accelerates the selection of the right path in the water maze also reducing the number of mistakes. Surgery ablation of pineal (pinealectomy), on the contrary, aggravates behavior disturbances in both tests. These data make it possible to say that MT possesses some nootropic features, and could be regarded as a potential cognitive enhancer [5].

This conclusion made following the experiment outcomes is in line with the results of observations which produced on human. Regular intake of MT (3mg)healthysubjects significantlyimproved of their memory that once suffered from psychoemotional stress [72]. Obviously, the hormone's mnemotropic properties determined by its direct impact on the brain structures' functions come along with improved perception. In particular, based on our data obtained through computerized campimetry, it reduces the photosensitivity thresholds in human retina, especially against the background of functional loss of vision [32].

Also, MT showed a capacity for easing various neurological disorders, such as seizures, consequences connected with cerebral trauma, and stroke. Anticonvulsant activity of MT can be seen from abundant experimental evidences obtained under various methodical conditions from various animals (mostly rodents) and using a wide range of MT dosages. For instance, when used in a pentylenetetrazol-induced convulsion model (guinea pigs; 50-160 mg/kg) MT increased the epileptoid responses threshold and prolonged their latent period. Chronic intake of MT (10-50 mg/ $\mathrm{kg}$ ) made it harder to provoke pilocarpine-induces convulsion in rats, as well as cortical epileptic afte rdischarges to repeated stimulation of amygdale; it can prevent electroconvulsion in case of both systemic and intraventricular use, etc. Yet, mention to be made that such preventive effect can be obtained from MT doses that are much higher than those used to improve behavior $[19,41,61,78]$.

The results of experiments involving human beings, even though not so obvious, often prove anti-convulsion features in MT. For instance, in child neurology the most responsive to it are various types of epilepsy and epileptoid convulsions typically associated with insomnia. Patients demonstrate reduced convulsive paroxysms, with lower intensity, and a trend towards better EEG and night sleep. MT often gave positive result when combined with traditional anti-convulsive pharmacotherapy (diphenylhydantoin, carbamazepine, etc.) and to overcome its resistance [12, 38, 74, 84].

MT also showed protective features in case of cerbral trauma consequences. Various animal models were used to demonstrate behavioral and neurological disorders as well as pathochemical shifts in the brain, which were similar to those found in people. The rodents that survived mechanical cerbral trauma or subarachnoid hematomas become disorganized in their activity when put to typical behavior tests, which comes together with specific muscle and vestibular disorders. Chronic (1-3 weeks) preliminary administration of MT (up to $10 \mathrm{mg} / \mathrm{kg}$ ) significantly reduced the mortality rate among the animals, as well as their behavior and neurological deficit that came together with brain trauma. At the same time its effect caused certain pathomorphological shifts such as limited zone of contusion, reduced brain edema, and facilitated reparation [17, 30, 39, 42, 75]. MT proved effective for acute cerebral circulation disorders of other genesis, in particular in case of stroke.

The fact that it has initially been part of the natural system for brain protection against ischemic lesion is supported with the outcomes of experiments where animals (through various ways - pineal extirpation, prolonged exposure to light) provoked the hormonal deficit. Such conditions make more prominent the behavior and morphological shifts produced by disorganization of cerebrovascular circulation, which may be determined, for instance, by occlusion of median cerebral artery or cortical photothrombosis in rats. On the other hand, injection of average MT doses (4-5 $\mathrm{mg} / \mathrm{kg}$ ) within the first 1-2 hours after the above-mentioned procedures limited the animals' brain infarction zone and the degree of its edema. The morphological features in various brain formations (neocortex, hippocampus, striatum) combined with the data from nucleic magnetic resonance serve evidences that pineal hormone has clear neuroprotective activity. Such data made it possible to recommend preventive treatment with MT to elderly patients especially demonstrating high risk of stroke [7, 28, 57, 71]. 
Besides, MT revealed therapeutic capacity for certain types of neurodegenerative brain pathology like Alzheimer's and Parkinson's diseases. Modern knowledge suggests that the leading pathogenetic factor in triggering Alzheimer's disease may be neurotoxic beta-amyloid peptide accumulated in rostral brain structures. In animal models local intracerebral injections of Abeta compound (the above-mentioned peptide's analogue) provoke severe cognitive disturbances similar to the dementia manifestations found in people suffering from the disease. Chronic treatment with MT (10$20 \mathrm{mg} / \mathrm{kg}$ ) in this case sometimes allows some reduction of the intensity in the mnestic disorders. Some (even though limited) clinical results can be achieved with MT administered to patients with Alzheimer's disease. After long-time injections (from a few weeks to several months) of high doses of the hormonal drug (typically above $10 \mathrm{mg}$ per day) the patients showed improved night sleep together with certain improvement in the cognitive functions [8].

Another variation of neurodegenerative pathology is parkinsonian syndrome including its specific motor phenomena and deficiencies in the affective and cognitive areas. The major role in parkinsonism development is attributed to disturbed interaction between the brain substantia nigra and basal ganglia. Here, an adequate experimental analogue of the disease is found in motor disorders and dementia developing in rodents in case of local injection of 6-oxidopamine neurotoxins or MPTP (a tetrahydropyridine derivative) into their substantia nigra, which is followed with nigrostriatal neurons degeneration. As shown in experiments performed in vivo and in vitro, local or systemic use of MT limits the death of substantia nigra cells and the degeneration of dopaminergic terminals in the corpus striatum, caused by the neurotoxins. Thus, it appears rather reasonable to employ MT to treat patients with Parkinson's disease. Unfortunately, despite the theoretical justification for such approach, the results of the pilot placebo-controlled tests (10 weeks, 5 or $50 \mathrm{mg}$ of MT per day) in clinical setting turned out to be not very much impressive. One of the reasons, just like in case with Alzheimer's disease could be the fact that neurodegenerative processes, when too advanced, may be difficultly treated with any medications [9].

\section{Cardiovascular disorders}

Along with brain activity, MT has an impact on the cardiovascular functions. A great number of respective experimental and clinical investigations projects have been carried out by now, and the outcomes of those have been joined into numerous reviews, so there shall be no need to quote some specific sources [24, 25, 27, 36, 51]. As shown presented below analysis, MT causes valuable therapeutic changes both in the arterial system, and in the heart performance.
Experiments involving healthy animals and perfusion of the isolated vessels obtained from them have produced rather controversial data on the vasoactive properties of MT. Some results suggest that it has the elevate of vessels tonus and also increases the blood pressure, while others contradict saying it may result in hypotension. However, experimental models of arterial hypertension produced more unidirectional changes. In spontaneously hypertensive rats (wide range of doses, $1-30 \mathrm{mg} /$ $\mathrm{kg}$ ) acute or chronic administration of MT resulted in stable decrease in the mean arterial pressure with no significant shifts in the heart frequency. In the same time it limits animal hypertension induced by electric shock stress. Contrary to that, removed pineal gland in rats leads to stable increase in the blood pressure while this schift could be effectively eliminated with exogenous MT treatment.

The conclusion drawn from experimental data on MT's antihypertensive properties coincide with the clinical results. A long-term observation over a large group of young women showed that reduction of plasma MT in them increased significantly the risk of essential hypertension. At the same time regular preventive administration of MT (1 mg per day) allowed preventing hormonal defect and pathology in whole. Repeated (for 4-6 weeks) use of MT alone (2 to $6 \mathrm{mg}$ daily before sleep) in patients with essential hypertension (I-II degree) brought down both systolic and diastolic pressure if compared to the controlled placebo group. Interesting to note that in subjects with a high nocturnal peak of plasma MT concentration also demonstrate much lower arterial pressure at those hours. Finally, a combination of MT with common antihypertensive drugs produces a more prominent clinical effect and at the same time restricting the side effects from the traditional therapy.

A similar trend manifested as insignificant or divergent influence on physiological functions in healthy humans and animals can be mentioned when investigated the cardiotropic peculiarities of MT. Thus, the variation of the heart rate in rats showed certain parasympathization of cardiointervalogram under low MT doses $(0.1 \mathrm{mg} / \mathrm{kg})$, which, however, did not progress yet went weak along with increased doses (up to 0.5 and $1 \mathrm{mg} / \mathrm{kg}$ ). A similarly unstable bradycardia was registered in monkeys (after injecting $0.2-0.4 \mathrm{mg} / \mathrm{kg}$ of MT). In young healthy women the bradycardic properties of MT (1 mg daily) can be detected only if they were divided into groups depending on their chronotypic features. On the other hand, pineal deficit (pinealectomy) aggravates the cardiac pathology in animals while injection of exogenous MT helps limit that.

Indeed, the heart rate disturbances and mortality among rats with myocardial ischemia/reperfusion went up once they had their pineal removed while preliminary MT injections $(0.4 \mathrm{mg} / \mathrm{kg})$ offered obvious protective effect. Its oral administration ( 2.5 or $5 \mathrm{mg} / \mathrm{kg}$ ) animals in post-ischemic reperfusion period was definitely positive for myocardial contractility and increased the cardiac output. The 
specificity of this action was confirmed with its attenuation with a selective MT receptor antagonist luzindole. The cardioprotective properties of MT were also shown in a rat model experimental infarction caused by ligation of coronary artery, and in isolated hearts. In the latter case its adding into the perfusion liqor not just weakened the intensity and duration of reperfusion arrhythmia and myocardium postischemic dysfunction but also induced coronary vasodilatation.

In view of the things mentioned above there appears an obvious reason behind the first MT clinical tests as monotherapy and in combination with specific drugs treating patients with cardiac disorders. Isolated and especially in combination with other drugs, MT (3 or $6 \mathrm{mg}$ ) would significantly increase the treatment effectiveness. This was seen from lesser heart rate disorders, reduced number of anginous fits and their duration, reduced need for nitroglycerine and higher tolerance to physical stress (see 30). Interestingly, there has been shown inverse relation between the class of coronary artery disease and the level of MT excreted in urine. Some data shows that patients with coronary pathology, if compared to healthy beings, lack common agebound dynamics of the hormone production. This explains the idea of using MT as prevention against sudden cardiac arrest in elderly peoples.

\section{Humoral disorders}

MT has universal modulator properties expand into the blood system as well. This hormone is capable of changing the functional features in various blood cells. In particular, based on in vitro and in vivo experiments, as well as on human investigations (less frequently) it showed therapeutic capacity related to normalization of thrombocyte homeostasis and erythrocyte protection against unfavorable conditions. There has already been sufficient reviewed information on both questions $[20,21]$, which makes any further reference excessive.

The effect that pineal gland and peripheral MT has on thrombocyte homeostasis perhaps should be named amongst its most important functional properties, given its direct involvement into thrombocyte activity. This, first of all, appears evident from the high density of the sites for specific binding of the hormone on the surface of blood plates, which attributes them to important blood transportation means supplying MT to tissues. On the other hand they do not just act as passive transporters but also are a source of extrapineal MT because thrombocytes possess major enzyme systems involved into MT synthesis.

Obviously, in people both thrombocyte and plasma (pineal) MT is directly involved into protection against thrombosis at night, and the major role here could be due to its capacity to limit the pro-aggregant properties in blood.

Indeed, as shown in vitro experiments on thrombocytes obtained from human blood, adding to them micromolar concentrations of MT inhibited (in a dose-dependent manner) their aggregation with a clear circadian rhythm. The effect was most obvious in the evening hours. The anti-aggregant effect covered both spontaneous and induced (with collagen, ADP) thrombocyte activity. Inhibited aggregation is accounted for by disturbances in the arachidonic acid cascade due to inhibited thromboxane production with no change from the prostacyclin part. In vivo these changes clearly correlated with the daily fluctuations in the hormone level in plasma. It should be noted that the peak of thrombocyte receptor sensitivity to MT preceded an increase of plasma MT level in blood. Hence, it is logic to view the natural constraint in pineal secretory activity, together with reduced production of the main hormone in the daytime, as one of the reasons behind the increased risk of thrombotic complications in patients with cardiovascular pathologies, which happens in the morning hours.

The MT anti-aggregant effects, obviously, are supplemented with its influence on blood coagulation and fibrinolysis. The results of experimental tests show that burning injury in rats results in disturbed blood coagulability with an increase in the prothrombine index, and a higher level of fibrinogen coming at the same time with disturbed thrombocyte morphology. Treatment of animals with MT $(10 \mathrm{mg} / \mathrm{kg})$ straight after the trauma weakened the described phenomena, also preventing disseminated intravascular blood coagulation. Similarly, a single used of MT by healthy people (3 mg) reduced the plasma level of fibrinogen and coagulation factor VIII, while the degree of coagulant activity clearly correlated with the hormone level in blood. Another close fact: MT administered to healthy volunteers following the same scheme prevented hyper-coagulation provoked by acute emotional stress.

Interesting that while maintaining the blood fluidity at a certain level, MT can at the same time stimulate thrombocytopoiesis. This fact is in perfect accordance with the presented here ideas on specific modulatory features found in the pineal hormone. For instance, in traumatic disease rat model, preliminary introduction of MT $(10 \mathrm{mg} / \mathrm{kg})$ restricted the thrombocytopenia intensity as well as reduced the intensity and duration of thrombocyte homeostasis disturbances. In the same time repeated use of high doses of MT (20 mg) in oncological patients weakened the hematological disorders induced by cytotoxic drugs. A similar protective effect manifested as thrombocytopoiesis was found in people against the background thrombocytopenia in case of lead-poisoning. Here we could also add reference to some cases of thrombocytopenic purpura where reliable results could be achieved only after long-term (three months) administration of MT.

Another evidence of universal therapeutic and adaptogenic properties of MT can be seen from observations made in the latest years, where it was proven to be necessary for maintaining the normal erythrocytes functioning. Involved in normal erythropoiesis and maintaining some important features 
in red blood cells (osmotic stability, deformability, filterability, etc.) it can find its demand in hematological practice in case of various experimental and clinical pathologies. Based on many research results the protective effect that the hormone has on erythrocytes is, first of all, due to restricted oxidative stress.

For instance, cholestasis in case of common bile duct ligation in rats or histamine-induced acute otitis in guinea pigs, will inevitably come with increased lipid peroxidation with reduced glutathione-peroxidase activity in erythrocytes happening along with accumulation of malonic dialdehyde. Given this background, preventive use of MT (5 or $10 \mathrm{mg} / \mathrm{kg}$ ) resulted each time in a reliable protective result with activated anti-oxidant enzymes. Just the same way protective capacity towards the oxidant status of erythrocytes was found in case of diabetes, which inevitably comes together with negative structural-functional shifts in the red blood cells with increased microviscosity and reversible aggregation. As MT has shown anti-diabetes features [14] these come in accord with restricted oxidative stress in erythrocytes. This conclusion based on experimental outcomes coincides with the observation results for patients suffering from Type 2 diabetes. A longterm ( 30 days) administration of MT ( $5 \mathrm{mg}$ daily) not only produced clinical improvement and normalization of carbohydrate homeostasis but at the same time ensured a reduction of the malonic dialdehyde in the patients' erythrocytes as well as superoxide dismutase activation. A similar phenomenon was seen in people suffering from essential arterial hypertension. Regular use of MT (5 mg, 2-3 weeks) produced an increased antioxidant status in their erythrocytes, which coincided with a growing efficiency of the respective hypotensive therapy. Similarly, restricted oxidant stress in erythrocytes can be seen when MT is administered to patients with neurological disorders and muscular dystrophy as well as to pulmonological patients with bronchial asthma or lung failure.

The described properties of MT can be well expanded onto the respective erythrocyte protection against various toxic effect. This has been justified repeatedly in numerous experiments where the role of toxic agents was attributed to various metal salts (lead, iron, cadmium, etc.), drugs (lidocaine, propofol), antibiotics, etc. In all cases, apart from rather a standard decrease of the tissue antioxidant status, there was a constantly registered increases in the erythrocyte hemolysis, which, when experiments done in vivo, came along with developing genotoxic defect and polychromatic erythrocytes appearing in blood together with their micronuclear forms.

Preliminary injection of MT $(10 \mathrm{mg} / \mathrm{kg})$ to poisoned animals typically weakened or prevented the development of the described symptoms of intoxication. The protective activity of MT went up when it was combined with other antioxidants (tocopherol, ascorbic acid). At the same time removed pineal gland increased the animals' symptoms of oxidant stress in peripheral tissues and erythrocytes. The gland transplantation from younger rats to older ones, on the contrary, restricted the age-related increased fluidity of erythrocytic membranes and the intensity of free-radical hemolysis of cells.

Important is the point that the MT antitoxic effect towards erythrocytes is accompanied with limited signs of toxic lesion in peripheral tissues. For instance, uranium poisoning provokes intense oxidant stress in rat erythrocytes together with lesion in the kidney tissue. MT (10 or $20 \mathrm{mg} / \mathrm{kg}$ ) while normalizing the activity of antioxidant enzymes in the blood cells, also reduced renal toxicity.

A brief analysis of the actual data offered in the items published in the recent decade and presented here makes it possible to draw the attention of clinical hematologists to MT seen as a potential drug for various blood disorders. This is also of interest because MT substances have extremely low toxicity and are rather safe.

\section{Oral cavity and gastrointestinal tract pathology}

The protective properties of MT have been repeatedly shown in a great number of research projects investigating various types of experimental and clinical pathology associated with disturbances certain parts of the digestive system. These include oral cavity issues, stomach and intestine ulcers, and pancreas secretory activity disorders.

The potential use of MT in stomatology has already been point of review in a number of items, so we will dwell only briefly on certain aspects here $[26,50,59,77]$. The physiological role of MT in the oral cavity, first of all, can be related to increasing secretion in salivary glands. This is seen from the fact that intravenous MT administration done to narcotized rats (but in relatively high doses of 5 or $25 \mathrm{mg} / \mathrm{kg}$ ) provoked increased salivation. On the contrary, insufficient MT due to pinealectomy will result in dry mouth in rats and mucosal ulceration, while daily MT injections ( $1 \mathrm{mg} / \mathrm{kg}$ ) will successfully eliminate such disturbances. The MT capacity to reduce inflammation is combined here with certain anti-infectious features (see below). In particular, repeated MT use (2.5 $\mathrm{mg}$ daily) in patients with oral herpes had a restrictive effect on the herpetic lesions in the oral mucosa.

Natural MT is also directly involved into teeth development, just like it is directly related to the development of the bony skeleton in general. An immune-histochemical analysis has shown that certain type of MT receptors can express ameloblasts, external dental epithelial cells, and tooth tissue odontoblasts. This way it can regulate the function of odontogenic tooth buds in animals and human beings. Maintaining a proper status of periodontium MT can also protect the oral cavity against toxic effects of polymers that are widely employed in orthopedic dentistry. Finally, MT is involved into restricting tumorous process 
and inhibited, for instance, the growth of oral epidermal carcinoma in an experiment.

MT involvement in the protection of stomach and intestinal mucosa against various pathological factors is one of the most developed and clinically reasoned aspects of its protective role at large. The obtained morden data, just like in the cases described above, has already been reviewed repeatedly [36, 43, 44, 48, 74]. Based on this it is obvious to conclude that MT administration (5 to $10 \mathrm{mg} / \mathrm{kg}$ ) into various animals with experimental stomach and intestinal ulcers of different origin will ensure a reliable protective effect. This is related to restricted secretion in gastric glands, reduced tone in the stomach and intestines smooth muscle, and increased regeneration in the mucosa. And vice versa, functional or surgical inactivation of pineal gland will significantly aggravate the entire representation of a gastrointestinal pathology.

The data is also well in accordance with numerous MT placebo-controlled tests in clinical setting. The therapeutic effect of the hormonal drug (5-6 mg per day, for 2-4 weeks) was proven in case of hyperacid gastritis, stomach and duodenal ulcer, and reflux disease. In these cases the result was especially clear for a combined administration of MT with omeprazol or anticholinergic drugs. Its use is also well grounded in case of colitis, including ulcerative colitis, and irritable bowel syndrome.

MT proved efficient also for various disturbances in the pancreas by restricting its both excretory and incretory disorders development, in particular acute pancreatitis and diabetes [13, 14, 49, 56, 62, 66]. For instance, in rat models of acute pancreatitis induced with caerulein or through vascular disturbances in ischemia/reperfusion of the gland, preliminary MT injections (introduced in high doses alone, 10 or $50 \mathrm{mg} / \mathrm{kg}$ ) inhibited the development of morphological destruction in pancreatic cells. At the same time the manifestations of oxidant stress and the production of proinflammatory cytokines went down. MT was able to improve the microelement composition in the damaged pancreas and brain tissue in animals affected by severe intoxication with organophosphorous compounds. It is obvious that the MT protective influence does not just reach the affected pancreas alone yet the neighboring parts involved into the pathology, liver among them. Thus, in rats with cerulean-induced pancreatitis preventive MT (20 mg/kg), together with improved anti-oxidant status, provoked similar changes in the liver tissue with recovered alanine transaminase activity. When developing an acute pancreatitis, which was done with various means, removed pineal glands would inevitably aggravate the biochemical and morphological disturbances in the animals' pancreas and liver.

The sum total of experimental and clinical observations makes it possible to suggest that MT possesses some antidiabetic properties as well. Streptozotocin is known to provoke carbohydrate disturbances in rats' metabolism, which are close to Type 1 diabetes in humans. Hyperglycemia in this case is combined with changed tolerance to carbohydrate stress, reduced glucokinase and glucose-6-phosphate dehydrogenase activity in the liver, and restricted antioxidant status in the body as a whole. Repeated preliminary administration of MT ( $5 \mathrm{mg} / \mathrm{kg}$, two weeks) prevents these disturbances. In the Goto-Kakizaki strain of rats (used to modeling of human type 2 diabetes), on the contrary, showed hyperinsulinemia and hyperlipidemia, as well as reduced insulin receptor tyrosine kinase activity. However in this case MT demonstrates a clear antidiabetic effect eliminating these disturbances. In vitro experiments support the conclusion on the anti-diabetic activity shown by MT. For instance, mice with alloxan diabetes inevitably show severe degenerative changes in the islets of Langerhans. In the event these animals were given preliminary MT $(0.15 \mathrm{mg} / \mathrm{kg}$, for two weeks), however, then the number and the structure of the beta-cells in them were nearly identical to the cell elements in intact mice.

The limited studies involving humans support the likelihood of MT's protective properties concerning diabetes. Thus, MT administration (for a month, 5 $\mathrm{mg}$ ) in patients with metabolic syndrome associated with type 2 diabetes, who have disturbances in the plasma MT level and insulin, resulted in an improved clinical picture involving positive changes in the oxidant status.

Even though the limited volume of this work would not let us pay much attention to the MT protective capacity, yet apart from the above-mentioned pathological conditions, modern literature holds description of the MT effect in many other cases including its oncostatic activity and capacity to influence a number of other pathologies.

\section{Anti-infectious activity}

In vivo and in vitro tests offer strong evidence in favor of protective capacity that MT has against various bacterial, viral, and parasitic infections.

The anti-bacterial activity of MT has been shown on various experimental models where one of the most popular ones is septic shock induced by a lipopolysaccharide introduced into small animals under the experiments. The injections provoked mice and rats' endotoxemia causing damage to many organs and systems (liver, kidneys, heart, etc.) as well as complex biochemical changes that serve evidence of activated immune system and increased production of proinflammatory enzymes. Bacterial shock quickly resulted in lethal outcome.

Previously or background of the lipopolysaccharide MT administration produced a reliable and stable protective effect - the death rate in the animals went down, the bacterial intoxication had lower intensity symptoms in the internal organs, while there was registered a tendency toward improved functioning in the immune system and restricted unfavorable biochemical 
manifestations. So even a single prophylactic injection of the hormonal drug within a wide dose range (10-60 $\mathrm{mg} / \mathrm{kg}$, intraperitoneally) can prevent the bacterial lesion of the liver, renal and lung tissue, heart muscle, improve the humoral reactivity in the vascular endothelium, improve the erythrocyte deformability and these shifts was even more pronounced when MT was used several hours after the lipopolysaccharide administration [65, 76 et al.].

Along with the lipopolysaccharide, to reproduce bacterial intoxication the researchers employed other models of septic shock, including ligation and injuries to rats' intestines, reproducing pyelonephritis in them, and introduction of zymosan. In these cases as well MT, when injected either at the onset of the infection or after several days, produced a clear protective effect. The same result was obtained when modeling coccal meningitis in rabbits. The interesting part is that antibacterial protection sometimes was obtained from lower dosages of the MT as well below $1 \mathrm{mg} / \mathrm{kg}$, while a combined use with an antibiotic would add to the efficiency [55 et al.].

The anti-infectious effects of MT were also observed from various models of experimental viral pathology. In rabbits suffering from the viral hemorrhagic disease (necrotic hepatitis) MT treatment (10 and $20 \mathrm{mg} / \mathrm{kg}$ ) at various periods after the infection reduced the death rate also inhibiting the apoptosis in the liver tissue. The protective effect that MT had on hepatocytes coincided with limited caspase-3 activity and lower expression of tumor necrosis factor, which came together with increased production of Bcl2 and Bcl-1L. Besides, the anti-apoptosis effect was accompanied with reduced expression of proinflammatory enzymes [79].

Similar results were obtained from other viral models reproduced in mice. In particular, their contagion with leukemia retrovirus caused a typical immunodeficiency syndrome, which manifested itself as inhibited cytokine release by $\mathrm{T}$-helpers, intense lipid peroxidation (LPO) in the liver, induced vitamin $\mathrm{E}$ deficit, as well as other biochemical shifts typical of human HIV-related deficiency. The use of MT, isolated or in combination with dehydroepiandrosterone, restricted the intensity of the described disturbances, and eliminated dysregulation in the cytokine system [58].

In mice infected with Venezuelan equine encephalitis MT $(0.5 \mathrm{mg} / \mathrm{kg})$ injected prior to the contagion and several days afterwards, reduced the mortality rate. This came along with weaker cerebral damage, decrease of lipid peroxidation products level in the brain tissue, and limited production of proinflammatory cytokines. When MT was used simultaneously with luzindole (MT receptor selective blocker) it reduced the protective action of the hormone drug thus stressing its specificity [68]. In vitro tests, too, showed viral infection to be weakened. While encephalitis virus introduced into the culture of neuroblastoma cells significantly increased LPO, further introduction of MT reversed the action [85].

There are facts witnessing that the MT protective effect does not just cover experimental manifestations of bacterial and viral infections, but also parasitic ones as well, including trypanosomes. When rat models reproducing various types of trypanosomiasis (African and American trypanosomiasis - Chagas disease) were treated with MT (preventive or within a week after the contagion; 5 or $10 \mathrm{mg} / \mathrm{kg}$ ) it improved the animals' general state in the acute phase of the disease restricting parasitemia seen through a reduced number of trypomastigotes in blood [73].

The results of the above-mentioned experiments appear to be sufficient to prove the capacity of exogenous MT to enforce the body against various pathogenic infections. Despite this, clinical practice, for obvious reasons lacks attempts at evaluating this point in case of its isolated use in patients with infections. The still insufficient observations from clinical specialists are mostly reduced to including MT into the traditional anti-infection treatment regimens.

One of the first studies of this kind was carried out when investigating sepsis in newborns. As it was shown, the group of patients that were administered peroral intake of MT (10 mg, twice, with an hour's interval) demonstrated no mortality and the perinatal adjustment went on better among them. The control group of babies, which followed traditional treatment alone without MT, showed a $30 \%$ mortality rate during the initial period after birth. Later on the reason for preventive use of the hormonal substance for septic patients (especially for those with septic shock) was proven positive not for newborns alone but for adults as well [40, 53].

The MT additive capacity was proven for other infectious pathologies as well. Patients with stomach and duodenal ulcer (complicated with Helicobacter pilory infection) demonstrated improved overall status and faster ulcer regeneration in cases where they had their traditional treatment with omeprazole supplemented with MT (twice a day, $5 \mathrm{mg}$ each intake) [17]. Just the same way this supplement as well as introducing some other antioxidants (tocopherol, ascorbic acid, etc.) into the traditional therapy for people with infected burn trauma reduced the likelihood of lethal outcome and improved the recovery [47].

Due to its capacity of intensifying antiviral protection, MT got its share of demand in comprehensive treatment for herpes and respiratory infection. Its repeated administration (2.5 or $3 \mathrm{mg}$ daily) combined with acyclovir showed a reliably lower rate of herpetic skin and mucosa lesion if compared to isolated use of special anti-viral drug; as far as respiratory issues were concerned, MT use resulted in fewer cases of inflammation getting chronic [63]. 
In view of the wide prevalence of Chagas disease in Latin America the antitrypanosomal properties of MT appear very relevant from the clinical stance. Using this substance helps restricting the acute stage in patients while in case of a chronic course it helps prevent the development of cardiomyopathy, which is done through higher doses [45]

\section{Anti-inflammatory activity}

The anti-infectious activity of MT is well complemented by its anti-inflammatory capacity. The latter comes out from numerous studies, which we have investigated in details and will try to present briefly below [see 29].

The modern experimental and clinical data allow detecting a rather large number of pathologies associated with inflammation where MT proves effective. Such conditions include inflammation of joints, internal organs (e.g., ulcerative colitis), peripheral nerves. Also special attention is to be paid to the possibility of synergistic interaction of the pineal hormone and the drugs including to the group of nonsteroidal anti-inflammatory drugs (NSAID).

Indeed, as some authors observed, a repeated (2-3 weeks) treatment with MT of patients with rheumatoid arthritis may be a perfect supplement to the basic therapy and add to the effect. Thus, with daily use (once per day, $3 \mathrm{mg}$, at night) the patients reported lower joint stiffness in the morning with fewer joints swelling and aching. The clinical improvement correlated with better nocturnal sleep and rearranged rhythm of cortisol daily secretion. Higher MT dosages $(10 \mathrm{mg})$ together with some restriction of rheumatoid arthritis symptoms, improved significantly the antioxidant status of the patients. There is also numerous data demonstrating the MT capacity (3-6 mg) to reduce various types of headaches. Both in people and animals it also restricted the ototoxic action of aminoglycoside antibiotics, improved the treatment of burns, and in case of longer-term use (6-12 months) helped patients with ulcerative colitis. At the same time there is evidence to the fact that in some cases MT can exacerbate rheumatoid arthritis in people and in experimental animal models.

Some experimental papers pointed at the capacity of MT to potentiate the pharmacological effect of certain NSAID. In particular, its resorbtive action (in rats intraperitoneal administration 0.5 and $1 \mathrm{mg} / \mathrm{kg}$ ), but not local use (20 or $40 \mu \mathrm{g}$ ) caused restriction of paw edema and vocalization in animals, which were due to direct application of the proinflammatory agent. If combined with indometacin in the same model, the pineal hormone enforced the anti-inflammatory and the antinociceptive effects of the nonnarcotic analgesic drug by nearly $25 \%$. Meloxicam (belongin to the group of oxicams) when administerd in a low dosage $(2.5 \mathrm{mg} / \mathrm{kg})$ changed no brain edema degree and behavioral disturbances in rats, which were a response to the occlusion of the median cerebral artery. However, when combined with melatonin (20 $\mathrm{mg} / \mathrm{kg}$ ), which under the circumstances showed only some weak protective capacity, the same subthreshold dose of meloxicam produced a clear protective effect.

In animals with oxidant lesion to liver tissue due to common bile duct ligation, acetylsalicylate and melatonin reduced the liver cell damage also bringing up their oxidant status (accumulation of glutathione and reduction of malonic dialdehyde level). This result was obtained with isolated use of the drugs at same time after their combined use observed more significant effect.

We have to admit that the MT additive capacity in relation to NSAID is more common in experimental practice and is not so prevalent in clinical practice. However, there are solid reasons for their combined use to treat neuralgic patients. Based on the analysis of literature that we have conducted earlier, MT shows clear analgetic properties, which should enjoy demand when treating pain syndromes of various origins [16]. Its isolated use turned efficient in patients with fibromyalgia, migrainous fits, various headaches - in other words issues where specific NSAID are administered. So it comes as no surprise that in cases of serious nocturnal headaches supplementing the indometacin- or lithium-based treatment regimens with MT increased their effect and reduced tolerance.

The interaction between MT and the pharmacological activity of NSAID is of peculiar agonistic-antagonistic nature. The point is that while it elevates the specific drug's activity, but in same time decreases the side effects. In case of acetylsalicylate-induced stomach ulcer in rats oral administration of MT $(2.5-10 \mathrm{mg} / \mathrm{kg}$ ) or of its precursor tryptophan limited the scale of damage wrought to the mucosa by the drug. A clearer protective effect was also shown in relation to ulcerative lesion of colon in animals, which was caused by acetylsalicylate, where MT was introduced repeatedly through parenteral using, though in a high dose $(100 \mathrm{mg} / \mathrm{kg})$. Its protective action was also detected for piroxicam- or indometacin-induces stomach mucosal ulceration in rats.

The experimental data coincide with those obtained from studies involving human. Long-term (11 days) preliminary administration of MT ( $5 \mathrm{mg}$ ), as endoscopic data suggest, prevented the ulcerative processes in the volunteers' stomachs that regular intake of aspirin ( 1.0 daily) was supposed to cause.

There is description of the protective features of MT towards drug-induced lesion to other internal organs - even a single injection of a high indometacin dose $(10 \mathrm{mg} / \mathrm{kg})$ provoked in starving rats toxic lesion of kidney parenchyma. Such side effect not observed if the animal received surprisingly low doses of MT $(0.75 \mathrm{mg} / \mathrm{kg})$ for one week before used of indometacin. Both in vivo and in vitro, paracetamol hepatotoxicity in mice was successfully prevented with MT $(10 \mathrm{mg} / \mathrm{kg})$. 
Interesting as it might seem, but while restricting the side effect of the anti-inflammation drug the pineal hormone never reduced its specific antipyretic and anti-inflammatory action.

Apart from the mechanisms described below, the reason behind MT anti-inflammatory effect as well as its synergic interaction with certain NSAID could be rather peculiar. This, perhaps, should imply MT's involvement into the arachidonic acid cascade also disturbing the prostaglandin synthesis. Not affecting the prostaglandin receptors themselves it clearly suppresses the function of the cyclooxygenase (COX-2) enzyme inducible isoform.

\section{Cellular and systemic mechanisms of MT protective properties}

The facts provided above create an impression that MT is capable of ensuring a rather universal type of protection for virtually any organ or tissue performing any physiological function. And the core of such universality must include similar, if not identical, cell and system mechanisms. All these taken together are aimed at correcting the deviations that come up in case a human or an animal is subject to pathology. This correction, of course should involve both the central (pineal) and peripheral (regional) MT.

MT protects cells in two ways: through specific receptors and via non-specific (non-receptor) pathways. In the first case we are talking about mobilization of various types of MT receptors that have a membrane and nuclear localization. These have been identified in virtually all organs and tissues and the functional meaning here is obviously determined by a need for universal modulation of any physiological processes. In particular, in the brain receptor mechanisms are responsible for MT involvement into the activity of the neurotransmitter systems and emotiogenic structures; in the vascular wall it regulates the development of vasoactive factors; in the myocardium it ensures cardioprotection; in the gastro-intestinal tract correction of the hydrochloric acid synthesis, while immune modulation is employed to keep control of tissue antitumor protection, etc. For MT biology it is important that the activation of the specific MT receptors is supplemented with its ability to easily penetrate through cell membranes interfering cytosol metabolism with no receptor involvement.

At the cell level the protective effect of MT must be accounted for by a set of reasons, where the top importance should be undoubtedly attributed to its antioxidant activity since the modern knowledge declares that a higher intensity of oxidation stress is determining for most pathologies associated with cell damage. The MT antioxidant effect, in turn, is due to a number of mechanisms. While penetrating into cells with no support from specific receptors it demonstrates a unique capacity to inactivate free oxygen and nitrogen radicals and acts like a sort of trap to them. Given this, MT is efficient in restricting the lipid peroxidation and offers universal protection of living cells against damage. MT performs its antioxidant protection not only due to free radicals neutralizing, yet also through increasing the body's antioxidant status at large, and exceeds by far any other antioxidants available, such as ascorbic acid and tocopherol. Its antioxidant mission includes intensifying the activity of antioxidant enzymes (pyruvatoxidase, catalase, superoxide dismutase), increasing the glutathione synthesis, and reducing the role of prooxidant enzymes [15, 33, 70, 82]. Obviously, there is an auxiliary therapeutically meaningful effect of MT's restriction imposed on oxidant stress - the ability to eliminate the mitochondrial dysfunction arising in case of a cardiovascular age-related neurodegenerative pathology. Acting rather selectively towards mitochondrial membranes MT acts as a regulator of their bio-energetic function [81].

The immunotropic activity of MT should be regarded as a therapeutic factor pertaining to both systemic and regional level at the same time. In the meantime the weak immune protection is a pathogenetic factor not for infectious, yet for a number of somatic diseases as well. Notable is that the immunotropic features of MT are often known for their modulatory nature revealed as intensified immunological responsiveness on the breakdown against its previous reduction and, contrary to that, the limits in the initial hyperactivity of the immune system. Hormonal control may be imposed on both the central organs managing its function, and the peripheral cell elements in the humoral and tissue parts of the immunity [23, 46, 69].

There are some more factors to be added to the mentioned points about the MT protective effect on the cell level. In the central nervous system and in the periphery the hormone demonstrates a clearly cut anti-inflammatory activity with disturbed proinflammatory cytokines production and increased strength of the connective tissue. It also restricts infectious inflammations, in part demonstrating anti-microbial activity, which made it effective for septic shock and as a nonsteroid anti-inflammatory agents synergist [60, $64,67]$. Besides, MT showed analgesic properties when used in human studies as well as in animal models. The cell mechanisms for the MT analgetic effect, which successfully complement the antiinflammation action, are determined with its inhibiting influence on the generation process and transmission of pain signals in the brain and at the peripheral pain receptors level [16, 37, 52, 80]. Finally, we cannot ignore its inhibitory effect on apoptosis with a simultaneous intensification of reparation in damaged tissues [66].

From the point of system mechanisms the leading role has to be attributed to the MT's cardinal property - to provide for the recovery, synchronization of biological rhythms of various frequencies, and the development, first of all, of a clearer circadian rhythmicity. This, perhaps is the basis for the ability of exogenous MT to cause hypnogenic 
effect and to eliminate latitude desynchronization $[6,86]$. The stabilization of rhythmic processes is one of the reasons for resistance to various pathogenic factors, as biorhythm disorganization accompanies any disorder; this stabilization is also part of the therapeutic effect in many pharmacologic drugs. The rhythm-stabilizing effect of MT may be determined by its inhibiting influence on the neurons of hypothalamus suprachiasmatic nuclei, which act as regulators of the daily fluctuations in many physiological functions. At the same time, the rhythm-driver and the pineal gland form very close morpho-functional connections, following our hypothesis, a particular chrono-biological block [3].

Another important element for any successful therapy of systemic nature, as we view it, should be the comprehensive effect that MT has on the higher nervous activity. The hormone has unique psycho-pharmacological activity profile is shaped by its simultaneous normalization of the sleep-wake system, emotional responsiveness, affective and cognitive spheres. Finally, this determines certain stabilization in the mental activity [11]. A combination with the rhythm organizing features of MT produces not only increased specific activity of psychotropic drugs of different classes, yet also an increased total resistance of the body, while if talking about people we could mention here a more positive subjective perception of overall well-being. It is obvious that a proper psycho-emotional attitude in patients has a role to play in determining the outcome of medication treatment for any pathology.

Apart from other features, pineal MT has shown a capacity to interfere the activity of peripheral endocrine glands. In a way, its global antistress effect can be seen from the modulation of adrenal cortex hormone production. As could be seen from our analysis of own and literary data, under pathological adrenocortical hyperactivity the foreground is taken by MT-mediated inhibitory influence of pineal gland on corticosteroid secretion, while this influence is typically masked in case of physiological norm [22].

\section{MT - a milti-purpose therapeutic drug?}

Based presented information about universal protective features of MT may be to suggest that as drug MT suitable for therapy any diseases. Or may be MT could be declared a panacea? However, we believe this kind of conclusion would be too hasty.

The thing is that the earlier numerous attempts aiming at giving a clinical evaluation to the theoretically justified capacity of MT sometimes failed. The probable reasons for the poorly pronounced effect of MT could include a whole

\section{References}

1. Anisimov V. N. Melatonin: role in the body, clinical use. Sanct Petersburg; 2007. 40 p.

2. Arushanian E. B. Role of pineal gland in brain protection against stress. Adv. Physiol. Sciences. 1996;27:31-50.

3. Arushanian E. B. Complex interrelations hypothalamic suprachiasmatic nuclei with pineal gland and striatum functionally single system for regulating daily fluctuations of behavior. J. Higher Nerv. Act. 1996;46:15-22. set of variable factors, which undoubtedly include improper dosing and incorrect timing (improper choice of the time of the day, season) for its intake, the initial endocrine status, etc. However, the most significant one, we believe, is neglecting the modulatory nature of the different effect that MT has.

Following the understanding of the natural biological role of the pineal gland its specific hormone a priori cannot (and, actually, should not) produce a prominent therapeutic effect. By the way, we have repeatedly postulated earlier that the main mission for pineal gland in the organism is adaptogenic adjustment of nearly all the human or animal functions to the altering environment, both external and internal. This gland in question belongs to cerebral and endocrine formations responsible for stationing homeostasis on the whole [6], so MT has no direct responsibility for any particular physiological process. The task for both the central (pineal) and the regional hormones is the correction only, in the event of any deviation from the norm. For this reason, as we think, exogenous MT typically has no impact on healthy organisms, which is often mistaken as an evidence of its lacking certain properties. In order to detect its pathophysiological mission and its therapeutic effect there is a need to have an initial borderline pathology.

On the other hand, based on the ideas of the protective nature of the modulating role the MT has we cannot expect significant results when employing the substance against a pathology that has advanced too far. In this case, for obvious reasons, prove effective (but not always) specific pharmacological agents. However we think that the ideas offered here should not serve grounds for any skeptic judgments concerning the results of experimental tests and clinical observations presented here.

The therapeutic possibilities of MT as we believe, should be demanded in complex pharmacotherapy of various brain and peripheral organ disorders. The potential success of this approach could be seen from our literature analysis that MT can potentiate the specific action of various drugs from different pharmacological groups [12]. Obviously, it is in such an adaptogenic and at the same time additive role this low-toxicity and relatively safe agent will attracted the attention of investigators and clinicists late, as a universal pharmacological modulator for limitation of different disturbed functions. But for confirmation such idea and its realization in clinical practice need randomized and placebo-controlled investigations in further.

4. Arushanian E. B. Antistress capacity of pineal melatonin. - Melatonin in norm and pathology. Moscow: «Medpractica»; 2004. P. 198-222.

5. Arushanian E. B. Pineal hormone melatonin - a new nootropic drug? Exp. and Clin. Pharmacol. 2005;68(3):74-79.

6. Arushanian E. B. Unique melatonin. Stavropol; 2006. 399 p.

7. Arushanian E. B. Protective role of melatonin in brain circulation disturbances. Rus. Med. J. 2010;18:57-61. 
8. Arushanian E. B. Melatonin and Alzheimer's disease. J. Neurol. and Psychiat. 2010;110:100-106.

9. Arushanian E. B. Hormonal drug melatonin for treating cognitive disorders in parkinsonism. Exp. and Clin. Pharmacol. 2010;73(3):35-39.

10. Arushanian E. B. Melatoninergic antidepressant Valdoxan. Exp. and Clin. Pharmacol. 2011;74(3):41-45.

11. Arushanian E. B. Melatonin - universal stabilizer of mental activity. J. Higher Nerv. Act. 2011;61:645-659.

12. Arushanian E. B. Pineal hormone melatonin in combined pharmacotherapy different brain disorders and somatic pathology. Exp. and Clin. Pharmacol. 2011;74(9):39-45.

13. Arushanian E. B. Protective role of melatonin in pancreas disorders. Exp. and Clin. Pharmacol. 2012;75(4):42-48.

14. Arushanian E. B. Melatonin and diabetes. - Melatonin: potential use in clinical practice. Moscow: «Imapress»; 2012. P. 30-39.

15. Arushanian E. B. Limitation oxidative stress as the main reason for universal protective features of melatonin. Exp. and Clin. Pharmacol. 2012;75(5):44-49.

16. Arushanian E. B. Analgesia and pineal hormone melatonin. Exp. and Clin. Pharmacol. 2012;75(8):44-48.

17. Arushanian E. B. Pregnancy and pineal gland. Rus. Newsl. Obstetr. and Gynecol. 2012;12(6):29-34.

18. Arushanian E. B. Importance of pineal hormone melatonin in pediatria and pediatric pharmacology. Med. Newsl. North Caucasus. 2012;1:116-122.

19. Arushanian E. B. Therapeutic capacity of pineal hormone melatonin in cerebral trauma. J. Neurol. and Psychiat. 2012;112(11):73-78

20. Arushanian E. B. Modern knowledge of circadian fluctuations in cardiovascular system, in norm and pathology. Clin. Med. 2012;4:11-18

21. Arushanian E. B. Experimental and clinical evidences to anti-convulsive activity of melatonin. J. Neurol. and Psychiat. 2013;113(11):112-116.

22. Arushanian E. B. Influence of melatonin on thrombocytes homeostasis and its circadian organization. Exp. and Clin. Pharmacol. 2013;76(5):32-38.

23. Arushanian E. B. Protective effect of melatonin on erythrocyte function. Med. Newsl. North Caucasus. 2013;8(3):95-100

24. Arushanian E. B. Pineal gland in pediatria: the role of pineal hormone melatonin in the genesis of autism. Med. Newsl. North Caucasus. 2013;8(2):82-85.

25. Arushanian E. B., Arushanian L. G., Elbekyan K. S. Place of pineal-adrenocortical relations in corrective regulation of behavior. Adv. Physiol. Sciences. 1993;24:73-79.

26. Arushanian E. B., Beyer E. V. Immunotropic features of pineal melatonin Exp. and Clin. Pharmacol. 2002;63(5):73-80.

27. Arushanian E. B., Beyer E. V. Brain pineal gland hormone melatonin and cardiovascular system. Part 1. Melatonin impact on blood pressure in norm and pathology. Med. Newsl. North Caucasus. 2011;1:69-73.

28. Arushanian E. B., Beyer E. V. Brain pineal gland hormone melatonin and cardiovascular system. Part 2. Melatonin impact on heart functions in norm and pathology. Med. Newsl. North Caucasus. 2011:2:90-95.

29. Arushanian E. B., Karakov K. G., Elbekyan K. S. Therapeutic effect of melatonin in oral cavity disorders. Exp. and Clin. Pharmacol. 2012;75(6):48-52.

30. Arushanian E. B., Mastyagina O. A. Melatonin in cardiovascular system and its pharmacological regulation. Exp. and Clin. Pharmacol. 2008;71(3):65-71.

31. Arushanian E. B., Naumov S. S. Stroke and pineal gland. J. Neurol. and Psychiat. 2009;109:67-74.

32. Arushanian E. B., Naumov S. S. Anti-inflammatory potential of melatonin. Clin. Med. 2013;7:18-22.

33. Arushanian E. B., Naumov S. S., Ponomareva V. A. Protective influence of melatonin on behavioral and morphological disorders caused by cerebral trauma in rats at different time of day. Exp. and Clin. Pharmacol. 2009;72(3):18-21.

34. Arushanian E. B., Ovanesov K. B. Role of pineal gland in pathogenesis of psychical depression. J. Higher Nerv. Act. 1991;41:822-827.
35. Arushanian E. B., Ovanesov K. B., Ovanesova I. M Comparative impact of melatonin and bilobil on photoreception and psycophysiological indices in patients with cerebral trauma. Exp. and Clin. Pharmacol. 2007;70(2):20-23.

36. Jerieva I. S., Volkova N. I. Nitrooxidative stress and its potential correction with melatonin. - Melatonin: potential use in clinical practice. Moscow: «Imapress»; 2012. P. $125-134$.

37. Kvetnoy I. M., Kvetnaya T. V., Raikhlin N. T. Extrapineal melatonin: place and role in homeostasis neuroendocrine regulation. - Melatonin in norm and pathology. Moscow: «Medpractica»; 2004. P. 34-47.

38. Malinovskaya N. K., Komarov F. I., Rapoport S. I. et al. Melatonin and cardiovascular system. - Melatonin in norm and pathology. Moscow: «Medpractica»; 2004 P. 85-101.

39. Malinovskaya N. K., Komarov F. I., Rapoport S. I. Melatonin in treating duodenal ulcer. - Melatonin in norm and pathology. Moscow: «Medpractica»; 2004. P. 146-162.

40. Ambriz-Tufuti M., Rocha-Gonzalez H., Cruz S. L. et al Melatonin: a hormone that modulates pain. Life Sci. 2009;84:489-498

41. Ardura J., Andres J., Gaemendia J. R. Melatonin in epilepsy and febrile seizures. J. Child. Neurol. 2010;25:888 891.

42. Ayer R. E., Sugawara T., Zhang J. H. Effects of melatonin in early brain injury following subarachnoid hemorrhage. Acta Neurochir. 2008;102(Suppl.):329-330.

43. Bagci S., Horoz O., Yidizdas D. et al. Melatonin status in pediatric intensive care patients with sepsis. Pediatr. Crit. Care Med. 2012;13:e120-e123.

44. Banach M., Gurdziel E., Jedrych M. Melatonin in experimental seizures and epilepsy. Pharmacol. Rep. 2011;63:1-11.

45. Bayir A., Kiresi D.A., Kara H. et al. The effects of mannitol and melatonin on $\mathrm{MRI}$ findings in an anima model of traumatic brain edema. Acta Neurol. Belg. 2008;108:149-154

46. Brzozowska P., Ptah-Belowska S., Pavlik M. et al. Mucosal strengthening activity of central and peripheral melatonin in the mechanism of gastric defense. J. Physiol. Pharmacol. 2009;60:47-56.

47. Bubenik G. A. Thirty four years since the discovery of gastrointestinal melatonin. J. Physiol. Pharmacol. 2008;59(Suppl. 2):33-51.

48. Cardinali D. P., Alvarez C. B. Melatonin in Chagas' disease Possible therapeutic value. Medicine. 2011;71:477-483.

49. Carrillo-Vico A., Reiter R. J., Lardone P. J. et al. The modulatory role of melatonin on immune responsiveness. Curr. Opin. Investig. Drugs. 2006;7:423-431.

50. Celinski K., Konturek P. C., Konturek S. J. et al. Effect of melatonin and tryptophan on healing of gastric and duodenal ulcers with Helicobacter pylori. J. Physiol. Pharmacol. 2011;62:521-526.

51. Choinacki C., Wisniewska-Jaroinska M., Walecka-Kapicaer et al. Evaluation of melatonin effectiveness in the adjuvant treatment of ulcerative colitis. J. Physiol. Pharmacol. 2011;62:327-334.

52. Col C., Dinler K., Hasdemir O. et al. Exogenous melatonin treatment reduces hepatocyte damage in rats with experimental acute pancreatitis. J. Hepatobilliary Pancreat. Sci. 2010;17:682-687.

53. Cutando A., Aneiros-Fernandez J. Aneiros-Cachaza J. Melatonin and cancer: current knowledge and its application to oral cavity tumors. J. Oral. Pathol. Med. 2011:38:164-175

54. Dominguez-Rodriguez A. Melatonin in cardiovascular disease. Expert. Opin. Investig. Drugs. 2012;21:15931596

55. Esposito E., Patriniti I., Mazzon E. et al. Melatonin reduces hyperalgesia associated with inflammation. J. Pineal Res. 2010;49:321-331.

56. Gitto E., Pellegrino S., Gitto P. et al. Oxidative stress of the newborn in the pre- and postnatal period and the clinical utility of melatonin. J. Pineal. Res. 2009;46:128-139. 
57. Hardeland R., Cardinali D. P., Srinivasan V. et al. Melatonin - a pleotropic, orchestrating regular molecule. Progr. Neurobiol. 2011;93:350-584.

58. Imamoglu M., Cay A., Cobanoglu K. et al. Effects of melatonin on suppression of renal scarring in experimental model of pyelonephritis. Urology. 2006;67:1315-1319.

59. Jaworek J., Szklarczyk J., Jaworek A. K. et al. Protective effect of melatonin on acute pancreatitis. Int. J. Inflam. 2012;76:1736-1775.

60. Kondoh T., Uneyama M., Nashino H. Melatonin reduces cerebral edema formation caused by transient forebrain ischemia in rats. Life Sci. 2002;72:583-590.

61. Kuehn C. C., Rodrigues Oliveira L., Santos C. D. et al. Melatonin and dehydroepiandrosterone combination: does this treatment exert a synergistic effect during experimental trypanosome cruzi infection? J. Pineal Res. 2009;47:253-259.

62. Kumasaka S., Shimozuma M., Kawamoto T. et al. Possible involvement of melatonin in tooth development: expression of melatonin 1a receptor in human and mouse tooth germs. Histochem. Cell. Biol. 2010;133:577-584.

63. Laliena A., San Miguel B., Crespo I. et al. Melatonin attenuates inflammation and promotes regeneration in rabbits with fulminant hepatitis of viral origin. J. Pineal Res. 2012;53:270-278.

64. Lima E., Cabral F. R., Cavalheiro E. A. et al. Melatonin administration after pilocarpine-induced status epilepticus: a new way to prevent or attenuate postlesion epilepsy? Epilepsy Behav. 2011;20:607-612.

65. Mulder H., Nagorny C. L., Lyssenko V. Melatonin receptors in pancreatic islets: good morning to a novel type 2 diabetes gene. Diabetologia. 2009;52:1240-1249.

66. Oda P. S., Rde P. Regression herpes viral infection symptoms using melatonin and SB-73: comparison with Acyclovir. J. Pineal Res. 2008;44:373-378.

67. Oliveira L. G., Kuehn C. C., Santos C. D. et al. Enhanced protection by melatonin and meloxicam combination in experimental infection by Trypanosoma cruzi. Parasite Immunol. 2010;32:245-251.

68. Ozdemir D., Uysal N., Turgyan K. et al. The effect of melatonin on endotoxemia-induced intestinal apoptosis and oxidative stress in infant rats. Intensive Care Med. 2007;33:511-516.

69. Peschke E. Melatonin, endocrine pancreas and diabetes. J. Pineal Res. 2008:44:26-40.

70. Radogna F., Diederich M, Ghibelli L. Melatonin: a pleotropic molecule regulating inflammation. Biochem. Pharmacol. 2010;80:1844-1852.

71. Reiter R. J. Melatonin: its sources, its message and the interpretation message. Adv. Pineal Res. 1989:165-173.

72. Reiter R. J., Calvo J. R., Karbownik M. et al. Melatonin and its relation to the immune system and inflammation. Ann. N.Y. Acad. Sci. 2000;917:376-386.

73. Reiter R. J., Tan D. X., Jou M. J. et al. Biogenic amines in the reduction of oxidative stress: melatonin and its metabolites. NeuroEndocrinol. Lett. 2008;29:391-398.
74. Reiter R. J., Tan D.-X., Leon J. et al. When melatonin gets of your nerves: its beneficial actions in experimental models of stroke. Exp. Biol. Med. 2005;230:104-117.

75. Rimmele U., Spilmann M., Wolf O. T. et al. Melatonin improves memory acquisition under stress. Psychopharmacology. 2009;202:663-672.

76. Santello F. H., Frare E. O., Caetano L. et al. Melatonin enhances pro-inflammatory cytokines level and protect against Chagas disease. J. Pineal Res. 2008;45:79-85.

77. Sanzez-Barselo E. J., Mediavilla H., Tan D.-X. Clinical uses of melatonin: evaluation of human trials. Curr. Med. Chem. 2010;17:2070-2096.

78. Sarrafzadeh A. S., Thomale U. W., Kroppenstedt S. N. Neuroprotective effect of melatonin on cortical impact injury in the rat. Acta Neurochir. 2000;142:1293-1299.

79. Shang Y., Xu S. P., Wie J. at al. Melatonin reduces acute lung injury in enditoxemic rats. Clin. Med. J. 2009;122:1388-1393.

80. Shimozuma M., Tokuyama R., Tatehara S. et al. Expression and cellular localization of melatonin-synthesizing enzymes in rat and human salivatory glands. Histochem. Cell. Biol. 2011;135:389-396.

81. Solmaz I., Gurkanlar D., Goksoy C. et al. Antiepileptic activity of melatonin in guinea pigs with pentylentetrazol-induced seizures. Neurol. Res. 2009;31:989-995.

82. Spreer A., Gerber J., Baake D. et al. Antiinflammatory but no neuroprotective effects of melatonin under clinical treatment conditions in rabbit models of bacterial meningitis. J. Neurosci. Res. 2006;89:1575-1579.

83. Srinivasan V., Pandi-Perumal S. R., Spence D. W. et al. Potential use of melatonergic drugs in analgesia: mechanisms of action. Brain Res. Bull. 2010;81:362-371.

84. Srinivasan V., Spence D. W., Pandi-Perumal S. R. et al. Melatonin in mitochondrial dysfunction and related disorders. Int. J. Alzheimer Dis. 2011;21:234-245.

85. Tousoulis D., Briasoulis A., Papageorgiou N. et al. Oxidative stress and endothelial function: therapeutic interventions. Recent Pat. Cardiovascular Drug Discov. 2011:6:103-114.

86. Tunon M. J., San Miguel B., Crespo J. et al. Melatonin attenuates apoptotic level damage in fulminant hepatic failure, induced by the rabbit hemorrhagic disease virus. J. Pineal Res. 2011;50:38-45.

87. Uberos J., Augustin-Morales M. O., Molina-Carbello A. et al. Normalization of the sleep wake pattern and melatonin and 6-sulphatoxy-melatonin levels after a therapeutic trial with melatonin in children with severe epilepsy. J. Pineal Res. 2011;50:192-196.

88. Valero N., Espina L. M., Mosquera J. Melatonin decreases nitric oxide production, inducible nitric oxidase synthases expression and lipid peroxidation induced by Venezuelan encephalitis equine virus in neuroblastoma cell cultures. Neurochem. Res. 2006;31:925-932.

89. Zawilska J. B., Skene D. J., Arendt J. Physiology and pharmacology of melatonin in relation to biological rhythms. Pharmacol. Rep. 2009;61:383-410. 